\title{
Noncontact Measurement of Charge Carrier Lifetime and Mobility in GaN Nanowires
}

\author{
Patrick Parkinson, ${ }^{* \dagger}$ Christopher Dodson, ${ }^{\ddagger}$ Hannah J. Joyce, ${ }^{\text {II }}$ Kris A. Bertness, ${ }^{\ddagger}$ Norman A. Sanford, ${ }^{\ddagger}$ \\ Laura M. Herz, ${ }^{\text {, }}$ and Michael B. Johnston*, II \\ ${ }^{\dagger}$ Electronic Materials Engineering, Research School of Physics and Engineering, The Australian National University, Canberra, ACT \\ 0200, Australia \\ ${ }^{\ddagger}$ Quantum Electronics and Photonics Division, National Institute of Standards and Technology, Boulder, Colorado, United States \\ ${ }^{\mathbb{I}}$ Department of Physics, Clarendon Laboratory, University of Oxford, Parks Road, Oxford OX1 3PU, United Kingdom
}

\section{Supporting Information}

ABSTRACT: The first noncontact photoconductivity measurements of gallium nitride nanowires (NWs) are presented, revealing a high crystallographic and optoelectronic quality achieved by use of catalyst-free molecular beam epitaxy. In comparison with bulk material, the NWs exhibit a long conductivity lifetime ( $>2 \mathrm{~ns})$ and a high mobility $(820 \pm 120$ $\left.\mathrm{cm}^{2} /(\mathrm{V} \mathrm{s})\right)$. This is due to the weak influence of surface traps with respect to other III-V semiconducting NWs and to the favorable crystalline structure of the NWs achieved via strainrelieved growth.

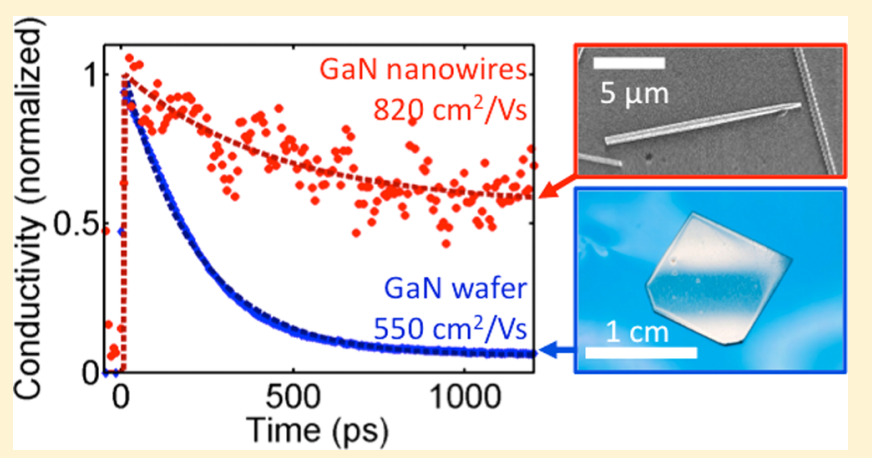

KEYWORDS: GaN, nanowire, photoconductivity, terahertz, surface plasmon

Cemiconductor nanowires (NWs) have been shown to have $\checkmark$ a multitude of practical applications throughout the fields of electronics and optoelectronics, ${ }^{1}$ for instance as nanoscale transistors, ${ }^{2}$ lasers, ${ }^{3}$ or in photovoltaic applications. ${ }^{4,5}$ Nanostructures such as NWs or nanoclusters are particularly attractive in the field of nanotechnology due to their applicability as interconnects or functional units in nanoelectronics. ${ }^{6}$ Gallium nitride $(\mathrm{GaN})$ is a prototypical highquality wide bandgap semiconductor, and it has been recently shown that NWs of GaN can be grown to an excellent crystallographic and electronic standard. ${ }^{7}$ Furthermore GaN nanowires are showing great promise for optoelectronic device applications; indeed low threshold lasing at room temperature has been observed from $200 \mathrm{~nm}$ diameter $\mathrm{GaN}$ nanowires. ${ }^{8}$ Using optical-pump terahertz-probe (OPTP) spectroscopy, we investigate the unique electronic properties of $\mathrm{GaN}$ nanowires arising from these high-quality growth conditions and material scale. This is an ideal technique for investigating nanostructured semiconductors, and couples the advantages of a noncontact method with ultrafast time resolution, as well as resolving the photoconductivity spectrum across an energy range identified with plasmonic and momentum scattering effects.

Gallium nitride is one of the most important wide-bandgap $\left(E_{\mathrm{g}} \approx 3.5 \mathrm{eV}^{10}\right)$ III-V semiconductors for electronic and optoelectronic applications. ${ }^{11}$ This is due primarily to its applicability for ultraviolet optoelectronics and high-power and high-frequency electronics. There have been several terahertzfrequency spectroscopic measurements on epitaxial GaN layers to date. However, these have focused upon equilibrium properties - i.e. intentionally or unintentionally doped samples. In 2003, Zhang and co-workers modeled the terahertzfrequency conductivity spectrum of $\mathrm{GaN}$ layers by use of the Drude model, extracting a carrier mobility of $\mu=1500 \mathrm{~cm}^{2} /$ (V.s). ${ }^{12}$ Mobility values ranging from $\mu=300$ to $1200 \mathrm{~cm}^{2} /$ (V.s) have been calculated by other groups using the Drude model at low temperature, ${ }^{13}$ using localized versions of the Drude model $^{14}$ and correcting for lattice vibrations. ${ }^{15}$ One possible reason for the large variation in measured mobility is the trap distribution for thin films; Oila et al. have shown that gallium vacancies preferentially form close to the interface with the substrate. Because of this, the sample thickness of epitaxially grown $\mathrm{GaN}$ has a large impact upon the measured electrical properties. $^{16}$

While devices based upon (planar epitaxial) GaN are now commonplace, the field of nanostructures of $\mathrm{GaN}$ is relatively new. ${ }^{17} \mathrm{GaN}$ NWs can now be grown to avoid many of the complications involved with thin-film and bulk material growth. These advantages arise from the nanowire morphology that provides a route for lattice relaxation and defect annihilation during growth. ${ }^{7,18,19}$ Additionally, while it is known that surface recombination routes play a large role in determining the

Received: May 21, 2012

Revised: August 14, 2012

Published: August 27, 2012 
carrier lifetime in some $\mathrm{III}-\mathrm{V} \mathrm{NWs},{ }^{20-22}$ this effect is much reduced in $\mathrm{GaN}^{23}$ The development of catalyst-free growth techniques that utilize molecular beam epitaxy (MBE) methods have been shown to provide NWs with very high material and optoelectronic quality, ${ }^{7,18,19}$ as required for integration into nanoelectronics. An important factor in this regard is the nanowire growth orientation whereby the polar $\langle 0001\rangle$ (c-axis) $\mathrm{GaN}$ NWs produced by MBE growth on $\mathrm{Si}(111)$ are remarkably free of defects and strain. ${ }^{7,19}$ On the other hand, nonpolar $\langle 11 \overline{2} 0\rangle$ ( $a$-axis) oriented NWs are often found to contain extended networks of defects and stacking faults. ${ }^{24}$

Analyses of field-effect electronic transport measurements in GaN NWs by various groups have yielded a fairly wide range of estimates for room-temperature electron drift mobility $\mu$, spanning roughly $60-650 \mathrm{~cm}^{2} /(\mathrm{V} \cdot \mathrm{s}) .{ }^{17,25-27}$ The proper interpretation of these measurements is often in dispute, because complications arise from such factors as electrical contacts, use of $c$-axis versus non- $c$-axis NWs, method of NW growth, accuracy and applicability of the simulation schemes employed, traps in gate oxides, and Fermi-level pinning at the NW surfaces, leading to depletion layers. Many of these issues were addressed and discussed by Mansfield and co-workers in their study of $c$-axis MBE-grown GaN NWs. ${ }^{28}$ More recently, Sanford et al. analyzed transient photoconductivity and dark conductivity of $c$-axis MBE grown GaN NWs and estimated $\mu$ to be in the range of $500-700 \mathrm{~cm}^{2} /(\mathrm{V} \cdot \mathrm{s}) ;^{29}$ and Talin et al. have estimated $\mu \approx 400 \mathrm{~cm}^{2} /(\mathrm{V} \cdot \mathrm{s})$ in MOCVD-grown $c$-axis GaN NWs by examining space-charge-limited conduction. ${ }^{30}$ Finally, it is interesting to note that the best Hall mobility for MBE GaN films grown on Si substrates is $800 \pm 120 \mathrm{~cm}^{2} /(\mathrm{V}$ s). ${ }^{31}$ This latter result is reasonably consistent with refs 29 and 30 if one assumes a conservative estimate for the Hall factor of roughly $1.2-1.3 .^{32}$ Comparison of the results reported in refs $29-31$ is relevant, because all three reports consider samples with free-carrier concentration in the range of low-to-mid $10^{16}$ $\mathrm{cm}^{-3}$.

To date, noncontact spectroscopy of GaN NWs has consisted primarily of temperature-dependent photoluminescence measurements, which revealed bulklike emission and a range of photoluminescence lifetimes from 200 ps to over $2 \mathrm{~ns}$ (at room-temperature). ${ }^{33}$ The NW geometry also permitted unambiguous measurement of the polarization anisotropy of the photoluminescence for GaN. ${ }^{34}$ Recently Tsokkou and coworkers performed room-temperature nondegenerate transient absorption on chemical vapor deposition (CVD)-grown GaN nanowires to reveal subnanosecond carrier decays routes via midgap and deep acceptor defects. ${ }^{35}$

In this Letter, we present the first time-resolved photoconductivity measurements performed on strain-free GaN nanowires. The photoinduced conductivity transient is shown to have a monoexponential form with a long room-temperature conductivity lifetime ( $>2 \mathrm{~ns})$, similar to that of the lowtemperature photoluminescence lifetime shown in ref 33 . In addition, the spectral conductivity is measured and modeled in the range $0.2-2.5 \mathrm{THz}(1-10 \mathrm{meV})$, providing a roomtemperature value for carrier mobility of $\mu=820 \pm 120 \mathrm{~cm}^{2} /$ $(\mathrm{V} \cdot \mathrm{s})$.

The GaN nanowire sample was prepared by use of molecular beam epitaxy (MBE) onto a silicon wafer, before transfer to a quartz substrate. Briefly, silicon-doped GaN NWs were grown by use of molecular-beam epitaxy onto a $\mathrm{Si}(111)$ substrate, ${ }^{7,36}$ producing NWs grown along the $c$-axis with side surfaces that conform to $m$-planes. The NWs were removed from the substrate by ultrasonication in isopropanol, followed by redeposition onto $z$-cut quartz. The nanowires were measured to have an n-type doping concentration of $1 \times 10^{17} \mathrm{~cm}^{-3}$ by use of Raman spectroscopy. ${ }^{37}$ A field-emission scanning-electron microscopy (FESEM) image of a typical sample is shown in Figure 1. The as-deposited NWs have mean diameters of 310

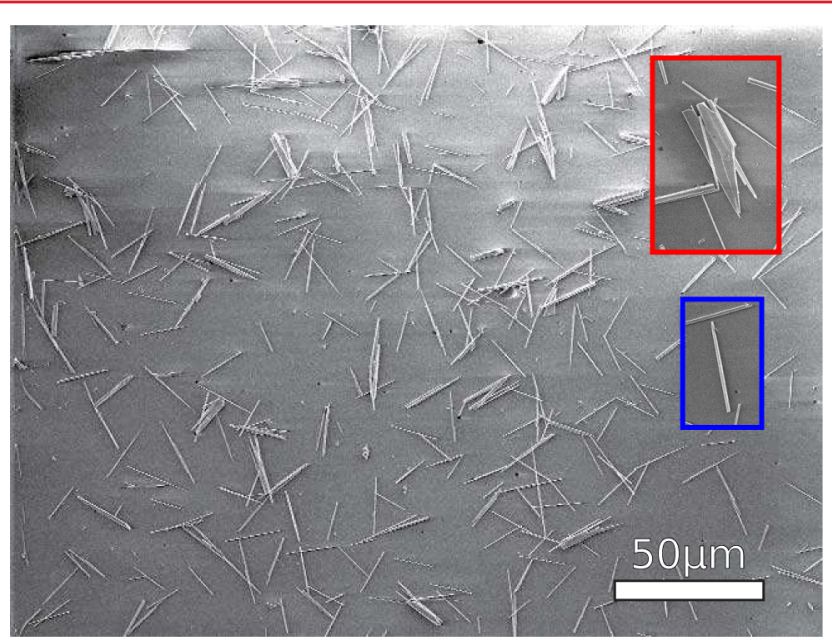

Figure 1. FESEM image of GaN NWs after redeposition onto a $z$-cut quartz substrate. The red rectangle (upper) shows an enlarged highlight of a large nanofin, while the blue rectangle (lower) shows an enlarged highlight of a $14 \mu \mathrm{m}$ long nanowire.

$\mathrm{nm}$ and lengths of $12.3 \mu \mathrm{m}$, measured by use of an ensemble of FESEM images. However, large and unstructured "nanofins" were also seen, which do not necessarily possess the same crystallographic orientation and standards as the NWs. Including these, the mean nanoparticle diameter and length increase to $484 \mathrm{~nm}$ and $12.4 \mu \mathrm{m}$, respectively. For comparison, the "bulk" GaN used was a $c$-plane, semi-insulating, $468 \mu \mathrm{m}$ thick sample purchased from Kyma Technologies, Inc. with a terahertz refractive index of $n=3 \pm 0.03$ and room-temperature absorption of $\alpha=50 \pm 5 \mathrm{~cm}^{-1}$ (see the Supporting Information). (Any mention of specific products or vedors does not constitute an endorsement by the National Institute of Standards and Technology.)

The photoconductivity lifetime and spectrum were measured by use of an optical-pump, terahertz-probe spectrometer similar to that described in ref 21 . Both the pump and probe pulses were split from an $810 \mathrm{~nm}, 40 \mathrm{fs}$ pulse generated in a Ti:Sapphire regenerative amplifier. The weak terahertz probe was generated using optical rectification in a $2 \mathrm{~mm}$ thick $\mathrm{ZnTe}$ crystal and had a peak electric field of $600 \mathrm{~V} / \mathrm{cm}$. The samples were photoexcited by using up to $10 \mathrm{~mJ} / \mathrm{cm}^{2}$ of excitation; in this way, photocarriers were injected via three-photon absorption. This process has previously been shown to result in photoluminescence characteristics that are identical to those observed upon above-gap (single-photon) excitation, ${ }^{33}$ and for this reason we consider the photoconductivity results to be the same as would be obtained from direct excitation. The sample was photoexcited at time $t=0$ by use of a $40 \mathrm{fs}$ pulse of $810 \mathrm{~nm}$ radiation, and the change in conductivity induced by this optical pulse was measured by use of a picosecond terahertz probe at later times with the delay set using an optical delay line. By use of the spectrometer in the fixed-gate mode, the time-resolved change in terahertz transmission was measured for periods up to $1.2 \mathrm{~ns}$ after excitation. The excitation pulse 
caused an increase in absorption of the terahertz probe; therefore the relative change in transmission $\Delta T / T$ is negative. This can be analytically converted to photoconductivity $\Delta \sigma$, which in turn is related to both the photocarrier density $N$ and mobility $\mu$ via $\Delta \sigma(\omega, \tau)=N(\tau) e \mu(\omega, N, \Gamma)$, where $\Gamma$ is the carrier momentum scattering rate. It is often reasonable to approximate the frequency-averaged mobility (and hence carrier mean scattering period) as a constant after the first few hundred femtoseconds, and in this case the photoconductivity decay curve is qualitatively the same as the photocarrier decay curve.

Figure 2 compares the photocarrier decay in GaN NWs to that observed for the bulk GaN sample. It can be seen that the

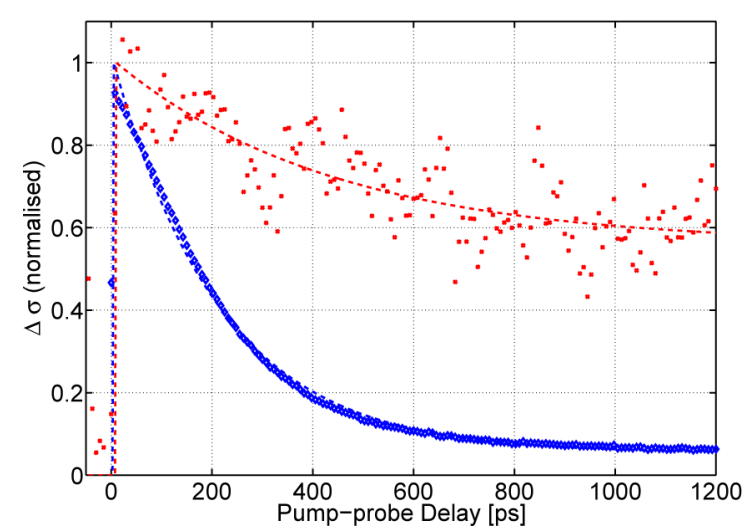

Figure 2. The normalized time-resolved photoconductivity of bulk GaN (blue dots and line) compared with GaN NW (red dots and line). Lines are a guide to the eye. The samples were excited at $810 \mathrm{~nm}$ with $9 \mathrm{~mJ} / \mathrm{cm}^{2}$.

carrier lifetime appears significantly longer for the NW sample than for the bulk sample with a $1 / e$ period of $\sim 2.5( \pm 0.5)$ ns. The GaN NW carrier lifetime determined by other techniques has typically been measured to be shorter; the lifetime observed for CVD-grown nanowires under direct excitation is $<0.65 \mathrm{~ns}^{35}$ More recently, Nguyen et al. have investigated charge recombination in room-temperature $\mathrm{InGaN} / \mathrm{GaN}$ dot-in-awire structures. ${ }^{38}$ They measured carrier lifetimes of a few nanoseconds and conclude that charge recombination is dominated by surface defects in a Shockley-Read-Hall process. Our results are in agreement with this conclusion, suggesting that surface passivation may be able to provide an even longer carrier lifetime. To determine the fluence dependence of the photoconductivity, a fixed-gate scan was taken for several pump-fluences (ranging from 0.1 to $10 \mathrm{~mJ} /$ $\mathrm{cm}^{2}$ ) for the bulk sample (see the Supporting Information). The significantly increased carrier lifetime observed in the NWs (under the same pump conditions) reveals an even lower carrier trap density, and the high-growth quality of MBE-grown GaN NWs.

By our using the spectrometer in the fixed-pump mode, the terahertz-frequency conductivity spectrum can be measured at a fixed delay after photoexcitation. Figure 3a shows the photoconductivity of the bulk GaN sample at 10 ps after photoexcitation (with a fluence of $0.33 \mathrm{~mJ} / \mathrm{cm}^{2}$ ) with fitting provided by the Drude model; a mobility value of $590 \pm 50$ $\mathrm{cm}^{2} /(\mathrm{V} \mathrm{s})$ was calculated, similar to that observed in ref 13 . Figure $3 \mathrm{~b}$ shows that the carrier population scales cubically with incident fluence for the bulk sample, which is consistent with a three-photon absorption process. Figure 3c,d shows spectral
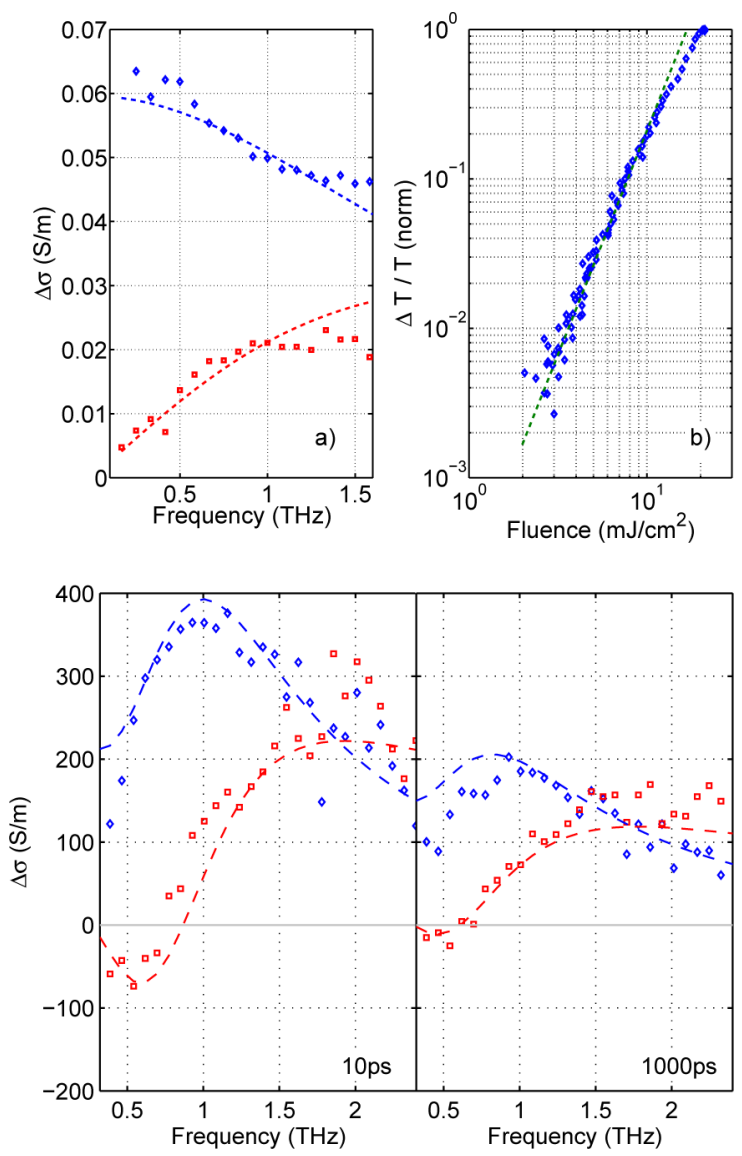

Figure 3. (a) The spectral conductivity of bulk GaN at 10 ps after excitation. A clear Drude-type response is observed corresponding to a mobility of $590 \mathrm{~cm}^{2} /(\mathrm{V} \cdot \mathrm{s})$. (b) The power dependence of the photoinduced absorption, revealing a clear cubic scaling. (Below) Terahertz spectral conductivity for the GaN NWs at (left) 10 ps and (right) $1000 \mathrm{ps}$ after photoexcitation with a fluence of $8 \mathrm{~mJ} / \mathrm{cm}^{2}$. The blue and red symbols (lines) represent the real and imaginary parts of the data (fit to the data), respectively. Note the plasmon-like peak, which is fitted to reveal a photoinduced carrier density of $3.4 \times 10^{16}$ $\mathrm{cm}^{-3}$ at $10 \mathrm{ps}$ and a carrier mobility of $820 \pm 120 \mathrm{~cm}^{2} /(\mathrm{V} \cdot \mathrm{s})$.

conductivity measurements at 10 and 1000 ps after excitation for the nanowire sample. This delay was chosen to avoid complications associated with temporal overlap of the pump and probe pulses. ${ }^{39}$ Neither the early- nor late-time conductivity shows a Drude-type response (as is observed for bulk $\mathrm{GaN}^{12,15}$ ) but instead a resonance-like peak is seen in both cases. Previously in nanoscale materials, this type of response has been attributed to excitonic or plasmonic absorption, or as a result of carrier localization in nanostructured materials (with models such as Drude-Smith, ${ }^{40}$ Cole-Davidson, ${ }^{41}$ and Kohlrausch $^{14}$ typically being used). We may exclude the response of excitons as the origin of the observed resonance; while OPTP spectroscopy is sensitive to exciton formation ${ }^{42}$ and stable excitons are expected to be found at room temperature, ${ }^{43}$ the exciton binding energy in GaN has been measured as $20-23 \mathrm{meV}(\sim 5 \mathrm{THz})^{11,43}$ and is therefore not expected to be seen in our measurements.

In order to convert terahertz transmission measurements of the NWs to conductivity spectra we followed the approach of ref 21. The conversion (detailed in the Supporting Information) takes into account both the limited interaction area (the NWs cover only $4 \%$ of the substrate), and the typical 
nanowire dimensions. Using this, the conductivity values given in Figure $3 \mathrm{c}, \mathrm{d}$ are obtained.

To fit these data, we use a modified Drude-plasmon model in which both the equilibrium conductivity and photoconductivity are composed of a Drude component and a component arising from a surface-plasmon resonance, given by $\Delta \sigma=\left(\Delta \sigma_{\text {Drude }}+\right.$ $\left.\Delta \sigma_{\text {Plasmon }}\right)$. The model follows that of ref 21 but with a minor modification due to the high background doping of the nanowires. Details of the model and a derivation of the fitting function are given in the Supporting Information. Briefly, the model uses four free parameters, $\Delta N$, the photocarrier density, $\rho$ and $\nu$, the distribution of equilibrium and photoinjected carriers (respectively) between Drude and Plasmon modes, and $\Gamma$, the carrier scattering rate.

The best-fit parameter values were calculated from a fit over the full $400 \mathrm{GHz}$ to $2.4 \mathrm{THz}$ range. By use of this fit, photocarrier densities of $3.4 \times 10^{16}$ and $1.8 \times 10^{16} \mathrm{~cm}^{-3}$ are calculated at 10 and 1000 ps, respectively. The carrier mobility may be calculated by use of the scattering rate obtained from this model, via the equation

$$
\mu=\frac{e}{m^{*} \Gamma}
$$

By use of eq 1 , (with $m^{*}=0.23 m_{\mathrm{e}}^{10,44,45}$ ) a carrier mobility of $820 \pm 120 \mathrm{~cm}^{2} /(\mathrm{V} \cdot \mathrm{s})$ is obtained.

In this paper, we have presented the first noncontact measurements of the electrical properties of gallium nitride nanowires. The optical-pump terahertz-probe technique provides an ideal tool for measuring the photocarrier lifetime and mobility in nanoscale materials. In comparison with a bulk $\mathrm{GaN}$ specimen, the carrier lifetime appears long, most likely due to the strain-free and defect-free growth that is the hallmark of $c$-axis oriented catalyst-free MBE GaN nanowire growth. The carrier lifetime is also long in comparison with those of other III-V nanowires due to the relatively low surface recombination velocity that is inherent to high-quality $\mathrm{GaN}^{25}$ We have extracted the room-temperature carrier mobility by modeling the terahertz-frequency photoconductivity spectrum with a Drude-plasmon model (rather than a strictly Drude model). This revealed a mobility of $820 \pm 120 \mathrm{~cm}^{2} /(\mathrm{V} \cdot \mathrm{s})$. For bulk GaN, we found that a Drude model was more applicable, which returned a value of $590 \pm 50 \mathrm{~cm}^{2} /(\mathrm{V} \cdot \mathrm{s})$. The result for bulk $\mathrm{GaN}$ is also reasonably consistent with the best Hall mobility reported for MBE-grown GaN films, when a conservative estimate for the Hall factor is used to scale the latter. These measurements show the advantages of noncontact conductivity and mobility measurements for nanoscale materials and reveal the high electrical quality of strain-free and defect-free growth of GaN nanowires.

\section{ASSOCIATED CONTENT}

\section{S Supporting Information}

The equilibrium terahertz absorption spectrum of bulk GaN, the power-dependent carrier lifetime in bulk GaN, and details of the function used to fit the terahertz absorption spectrum of $\mathrm{GaN}$ nanowires. This material is available free of charge via the Internet at http://pubs.acs.org.

\section{AUTHOR INFORMATION}

\section{Corresponding Author}

*E-mail: (P.P.) patrick.parkinson@anu.edu.au; (M.B.J.) m. johnston@physics.ox.ac.uk.

\section{Notes}

The authors declare no competing financial interest.

\section{ACKNOWLEDGMENTS}

The authors acknowledge the EPSRC [H.J.J., L.M.H., M.B.J.] and the Australian Reasearch Council [P.P.] for funding.

\section{REFERENCES}

(1) Li, Y.; Qian, F.; Xiang, J.; Lieber, C. M. Mater. Today 2006, 9, 18.

(2) Cui, Y.; Zhong, Z. H.; Wang, D. L.; Wang, W. U.; Lieber, C. M. Nano Lett. 2003, 3, 149.

(3) Duan, X. F.; Huang, Y.; Agarwal, R.; Lieber, C. M. Nature 2003, 421, 241.

(4) Tian, B.; Kempa, T. J.; Lieber, C. M. Chem. Soc. Rev. 2009, 38, $16-24$.

(5) Law, M.; Greene, L. E.; Johnson, J. C.; Saykally, R.; Yang, P. D. Nat. Mater. 2005, 4, 455-459.

(6) Xia, Y. N.; Yang, P. D.; Sun, Y. G.; Wu, Y. Y.; Mayers, B.; Gates, B.; Yin, Y. D.; Kim, F.; Yan, Y. Q. Adv. Mater. 2003, 15, 353-389.

(7) Bertness, K. A.; Sanford, N. A.; Barker, J. M.; Schlager, J. B.; Roshko, A.; Davydov, A. V.; Levin, I. J. Electron. Mater. 2006, 35, 576580.

(8) Gradečak; Qian, F.; Li, Y.; Park, H.; Lieber, C. M. Appl. Phys. Lett. 2005, 87, 173111.

(9) Merchant, S. K. E.; Lloyd-Hughes, J.; Sirbu, L.; Tiginyanu, I. M.; Parkinson, P.; Herz, L. M.; Johnston, M. B. Nanotechnology 2008, 19, 395704.

(10) Ilegems, M.; Montgomery, H. C. J. Phys. Chem. Solids 1973, 34, 885-895.

(11) Mohammad, S. N.; Salvador, A. A.; Morkoç, H. Proc. IEEE 1995, 83, 1306-1355.

(12) Zhang, W.; Azad, A. K.; Grischkowsky, D. Appl. Phys. Lett. 2003, $82,2841-2843$

(13) Nagashima, T.; Takata, K.; Nashima, S.; Harima, H.; Hangyo, M. Jpn. J. Appl. Phys., Part 1 2005, 44, 926-931.

(14) Tsai, T. R.; Chen, S. J.; Chang, C. F.; Hsu, S. H.; Lin, T. Y.; Chi, C. C. Opt. Express 2006, 14, 4898-4907.

(15) Guo, H. C.; Zhang, X. H.; Liu, W.; Yong, A. M.; Tang, S. H. J. Appl. Phys. 2009, 106, 063104.

(16) Oila, J.; Kivioja, J.; Ranki, V.; Saarinen, K.; Look, D. C.; Molnar, R. J.; Park, S. S.; Lee, S. K.; Han, J. Y. Appl. Phys. Lett. 2003, 82, 34333435.

(17) Huang, Y.; Duan, X. F.; Cui, Y.; Lieber, C. M. Nano Lett. 2002, 2, 101-104.

(18) Calleja, E.; Sanchez-garcia, M. A.; Sanchez, F. J.; Calle, F.; Naranjo, F. B.; Munoz, E.; Jahn, U.; Ploog, K. Phys. Rev. B 2000, 62, $16826-16834$.

(19) Cerutti, L.; Ristic, J.; Fernandez-garrido, S.; Calleja, E.; Trampert, A.; Ploog, K. H.; Lazic, S.; Calleja, J. M. Appl. Phys. Lett. 2006, 88, 213114.

(20) Prasankumar, R. P.; Choi, S.; Trugman, S. A.; Picraux, S. T.; Taylor, A. J. Nano Lett. 2008, 8, 1619-1624.

(21) Parkinson, P.; Lloyd-Hughes, J.; Gao, Q.; Tan, H. H.; Jagadish, C.; Johnston, M. B.; Herz, L. M. Nano Lett. 2007, 7, 2162-2165.

(22) Parkinson, P.; Joyce, H. J.; Gao, Q.; Tan, H. H.; Zhang, X.; Zou, J.; Jagadish, C.; Herz, L. M.; Johnston, M. B. Nano Lett. 2009, 9, $3349-3353$

(23) Dhesi, S. S.; Stagarescu, C. B.; Smith, K. E.; Doppalapudi, D.; Singh, R; Moustakas, T. D. Phys. Rev. B 1997, 56, 10271-10275.

(24) Tham, D.; Nam, C. Y.; Fischer, J. E. Adv. Funct. Mater. 2006, 16, 1197-1202.

(25) Motayed, A.; Davydov, A. V.; Mohammad, S. N.; Melngailis, J. J. Appl. Phys. 2008, 104, 024302.

(26) Gurwitz, R.; Shalish, I. Nanotechnology 2011, 22, 435705.

(27) Talin, A. A.; Leonard, F.; Katzenmeyer, A. M.; Swartzentruber, B. S.; Picraux, S. T.; Toimil-molares, M. E.; Cederberg, J. G.; Wang, X.; Hersee, S. D.; Rishinaramangalum, A. Semicond. Sci. Technol. 2010, 25, 024015 . 
(28) Mansfield, L.; Bertness, K.; Blanchard, P.; Harvey, T.; Sanders, A.; Sanford, N. J. Electron. Mater. 2009, 38, 495-504.

(29) Sanford, N. A.; Blanchard, P. T.; Bertness, K. A.; Mansfield, L.; Schlager, J. B.; Sanders, A. W.; Roshko, A.; Burton, B. B.; George, S. M. J. Appl. Phys. 2010, 107, 034318.

(30) Talin, A. A.; Swartzentruber, B. S.; Leonard, F.; Wang, X.; Hersee, S. D. J. Vac. Sci. Technol. B 2009, 27, 2040-2043.

(31) Nikishin, S.; Kipshidze, G.; Kuryatkov, V.; Choi, K.; Gherasoiu, I.; Zubrilov, A.; Tretyakov, V.; Copeland, K.; Prokofyeva, T.; Holtz, M.; Asomoza, R.; Kudryavtsev, Y.; Temkin, H. J. Vac. Sci. Technol. B 2001, 19, 1409-1412.

(32) Morkoç, H. Nitride Semiconductors and Devices; Springer-Verlag: New York, 1999.

(33) Schlager, J. B.; Bertness, K. A.; Blanchard, P. T.; Robins, L. H.; Roshko, A.; Sanford, N. A. J. Appl. Phys. 2008, 103, 124309.

(34) Schlager, J. B.; Sanford, N. A.; Bertness, K. A.; Barker, J. M.; Roshko, A.; Blanchard, P. T. Appl. Phys. Lett. 2006, 88, 213106.

(35) Tsokkou, D.; Othonos, A.; Zervos, M. J. Appl. Phys. 2009, 106, 054311.

(36) Bertness, K. A.; Roshko, A.; Mansfield, L. M.; Harvey, T. E.; Sanford, N. A. J. Cryst. Growth 2008, 310, 3154-3158.

(37) Robins, L. H.; Sanford, N. A.; Bertness, K. A.; Schlager, J. B. Raman Measurements of Carrier Density in n-type GaN Nanowires: Bulk and Surface Optical Phonon Modes, 2012 Materials Research Society Spring Meeting, San Francisco, CA, April 9-13, 2012.

(38) Hieu, P. T. N.; Djavid, M.; Cui, K.; Mi, Z. T. Nanotechnology 2012, 23, 194012.

(39) Kužel, P.; Kadlec, F.; Nčmec, H. J. Chem. Phys. 2007, 127, 024506.

(40) Smith, N. V. Phys. Rev. B 2001, 64, 155106.

(41) Davidson, D. W.; Cole, R. H. J. Chem. Phys. 1951, 19, 14841490.

(42) Kaindl, R. A.; Carnahan, M. A.; Hägele, D.; Lövenich, R.; Chemla, D. S. Nature 2003, 423, 734-738.

(43) Muth, J. F.; Lee, J. H.; Shmagin, I. K.; Kolbas, R. M.; Casey, H. C.; Keller, B. P.; Mishra, U. K.; Denbaars, S. P. Appl. Phys. Lett. 1997, $71,2572-2574$.

(44) Witowski, A. M.; Pakula, K.; Baranowski, J. M.; Sadowski, M. L.; Wyder, P. Appl. Phys. Lett. 1999, 75, 4154-4155.

(45) Meyer, B. K.; Volm, D.; Graber, A.; Alt, H. C.; Detchprohm, T.; Amano, A.; Akasaki, I. Solid State Commun. 1995, 95, 597-600. 